\title{
Habitable zones for Earth-mass planets in multiple planetary systems
}

\author{
Jianghui $\mathrm{JI}^{1}$, Lin LIU ${ }^{2}$, Hiroshi KINOSHITA ${ }^{3}$ and Guangyu LI ${ }^{1}$ \\ ${ }^{1}$ Purple Mountain Observatory, Chinese Academy of Sciences, Nanjing 210008, China \\ email: jijh@pmo.ac.cn \\ ${ }^{2}$ Department of Astronomy, Nanjing University, Nanjing 210093, China \\ email: xhliao@nju.edu.cn \\ ${ }^{3}$ National Astronomical Observatory, Mitaka, Tokyo 181-8588, Japan
}

\begin{abstract}
We perform numerical simulations to study the Habitable zones (HZs) and dynamical structure for Earth-mass planets in multiple planetary systems. For example, in the HD 69830 system, we extensively explore the planetary configuration of three Neptune-mass companions with one massive terrestrial planet residing in $0.07 \mathrm{AU} \leqslant a \leqslant 1.20 \mathrm{AU}$, to examine the asteroid structure in this system. We underline that there are stable zones of at least $10^{5} \mathrm{yr}$ for low-mass terrestrial planets locating between 0.3 and $0.5 \mathrm{AU}$, and 0.8 and $1.2 \mathrm{AU}$ with final eccentricities of $e<0.20$. Moreover, we also find that the accumulation or depletion of the asteroid belt are also shaped by orbital resonances of the outer planets, for example, the asteroidal gaps at $2: 1$ and 3:2 mean motion resonances (MMRs) with Planet C, and 5:2 and 1:2 MMRs with Planet D. In a dynamical sense, the proper candidate regions for the existence of the potential terrestrial planets or $\mathrm{HZs}$ are $0.35 \mathrm{AU}<a<0.50 \mathrm{AU}$, and $0.80 \mathrm{AU}<a<1.00 \mathrm{AU}$ for relatively low eccentricities, which makes sense to have the possible asteroidal structure in this system.
\end{abstract}

Keywords. methods: $n$-body simulations-planetary systems-stars:individual (HD69830, 47 UMa)

\section{Introduction}

To date, over 260 extrasolar planets have been discovered around the nearby stars within 200 pc (Butler et al. 2006; The Extrasolar Planets Encyclopaedia†) mostly by the measurements of Doppler surveys and transiting techniques. The increasing numbers of known extrasolar planets are largely attributed to increasing precision in measurement techniques. Observational improvements will likely lead to more substantial discoveries, including: (1)diverse multi-planetary systems, of which more than 20 multiple systems with orbital resonance or secular interactions are already known;(2)low-mass companions around main-sequence stars (so-called super-Earths), e.g., 55 Cancri (McArthur et al. 2004), GJ 876 (Rivera et al. 2005), HD 160691 (Santos et al. 2004; Gozdziewski et al. 2007); (3)a true Solar System analog, with several terrestrial planets, asteroidal structure and a dynamical environment consistent with terrestrial planets in the Habitable Zone (HZ) (Kasting et al. 1993) that could permit the development of life, e.g., Gl 581 (von Bloh et al. 2007); (4)a comprehensive census of a diversity of planetary systems, which will provide abundant clues for theorists to more accurately model planetary formation processes (Ida \& Lin 2004; Boss 2006).

Lovis et al. (2006) (hereafter Paper I) reported the discovery of an interesting system of three Neptune-mass planets orbiting about HD 69830 through high precision measurements with the HARPS spectrograph at La Silla, Chile. The nearby star HD 69830 is of spectral type $\mathrm{K} 0 \mathrm{~V}$ with an estimated mass of $0.86 \pm 0.03 M_{\odot}$ and a total luminosity

$\dagger$ As of Nov. 8, 2007, see http://exoplanet.eu/catalog.php and http://exoplanets.org/ 

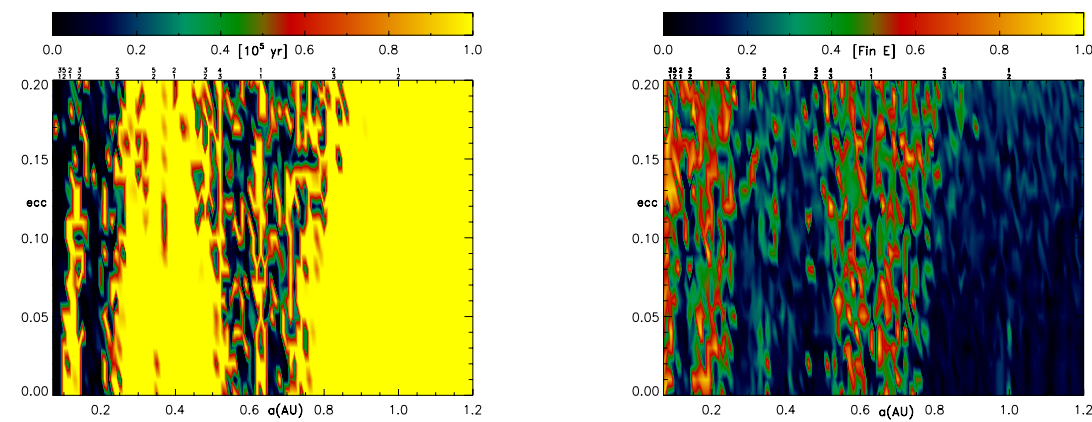

Figure 1. Left panel: Contour of the surviving time for Earth-like planets for the integration of $10^{5}$ yr. Right panel: Status of their final eccentricities. Horizontal and vertical axes are the initial a and e. Stable zones for the low-mass planets in the region between 0.3 and $0.5 \mathrm{AU}$, and 0.8 and $1.2 \mathrm{AU}$ with final low eccentricities.

of $0.60 \pm 0.03 L_{\odot}$ (Paper I), about $12.6 \mathrm{pc}$ away from the Sun. In addition, Beichman et al. (2005) announced the detection of a large infrared excess owing to hot grains of crystalline silicates orbiting the star HD 69830 and inferred that there could be a massive asteroid within 1 AU. Subsequently, Alibert et al. (2006) and Paper I performed lots of calculations to simulate the system and revealed that the innermost planet may possess a rocky core surrounded by a tiny gaseous envelope. This planet probably formed inside the ice line in the beginning, whereas the two outer companions formed outside the ice line from a rocky embryo and then accreted the water and gas onto the envelope in the subsequent formation process. Hence, it is important for one to understand the dynamical structure in the final assemblage of the planetary system (Asghari et al. 2004; Ji et al. 2005), and to investigate suitable HZs for life-bearing terrestrial planets (Jones et al. 2005; Raymond et al. 2006; von Bloh et al. 2007; Gaidos et al. 2007) advancing the space missions (such as CoRot, Kepler and TPF) aiming at detecting them, thus one of our goals is to focus on the issues of the potential Earth-mass planets in the system.

\section{Dynamical Structure and Habitable Zones in HD 69830 system}

Modern observations by Spitzer and HST indicate that circumstellar debris disks (e.g., $\mathrm{AU}$ Mic and $\beta \mathrm{Pic}$ ) are quite common in the early planetary formation. Beichman et al. (2006) used Spitzer to show that $13 \pm 3 \%$ of mature main sequence stars exhibit Kuiper Belt analogs. They further point out that the existence of debris disks is extremely important for the resulting detection of individual planets, and related to the formation and evolution of planetary systems. As mentioned previously, Beichman et al. (2005) also provide clear evidence of the presence of the disk in HD 69830. Subsequently Paper I's best-fit orbital solutions were for three Neptune-mass planets with well-separated nearlycircular orbits, which may imply that the HD69830 system is similar to our Solar System in that it is dynamically consistent with the possible presence of terrestrial planets and asteroidal and Kuiper belt structures. Hence, it deserves to make a detailed investigation from a numerical perspective.

To investigate the dynamical structure and potential HZs in this system, we performed additional simulations with HD69830's three Neptune-mass companions in coplanar orbits, and one massive Earth-like planet. In the runs, the mass of the assumed terrestrial planet ranges from $0.01 M_{\oplus}$ to $1 M_{\oplus}$. The initial orbital parameters are as follows: the numerical investigations were carried out in $[a, e]$ parameter space by direct integrations, 
and for a uniform grid of $0.01 \mathrm{AU}$ in semi-major axis (0.07 $\mathrm{AU} \leqslant a \leqslant 1.20 \mathrm{AU})$ and 0.01 in eccentricity $(0.0 \leqslant e \leqslant 0.20)$, the inclinations are $0^{0}<I<5^{0}$, and the angles of the nodal longitude, the argument of periastron, and the mean anomaly are randomly distributed between $0^{0}$ and $360^{\circ}$ for each orbit, then each terrestrial mass body was numerically integrated with three Neptune-mass planets in the HD 69830 system. In total, about 2400 simulations were exhaustively run for typical integration time spans from $10^{5}$ to $10^{6}$ yr (about $10^{6}-10^{7}$ times the orbital period of the innermost planet) (see also Ji et al. 2007 for details).

Figure 1 shows the contours of the survival time for Earth-like planets (left panel) and the status of their final eccentricities (right panel) for the integration over $10^{5} \mathrm{yr}$, where horizontal and vertical axes are the initial $a$ and $e$. The left panel displays that there are stable zones for a terrestrial planet in the regime between 0.3 and $0.5 \mathrm{AU}$, and 0.8 and 1.2 AU with final eccentricities of $e<0.20$. Obviously, unstable zones exist near the orbits of the three Neptune-mass planets where the planetary embryos have short dynamical survival time, and their eccentricities can quickly be pumped up to a high value $\sim 0.9$ (right panel). In these regions the evolution is insensitive to the initial masses. The terrestrial bodies are related to many of the mean motion resonances of the Neptunian planets and the overlapping resonance mechanism (Murray \& Dermott 1999) can reveal their chaotic behaviors of being ejected from the system in short dynamical lifetime. Furthermore, most of terrestrial orbits are within $3 R_{\text {hill }}$ sphere of the Neptune-mass planets, and others are involved in the secular resonance with two inner companions.

Analogous to our Solar system, if we consider the middle planet (HD 69830 c) as the counterpart as Jupiter, we will have the regions of mean motion resonances: 2:1 (0.117 AU), 3:2 (0.142 AU), 3:1 (0.089 AU) and 5:2 (0.101 AU), 2:3 (0.244 AU). In Fig. 1, we notice there indeed exist the apparent asteroidal gaps about or within the above MMRs (e.g., 3:1 and 5:2 MMRs), while in the region between 0.10 AU and 0.14 AU for $e<0.10$, there are stable islands where the planetary embryos can last at least $10^{5} \mathrm{yr}$. In addition, for Planet $\mathrm{D}$, most of the terrestrial planets in $0.50 \mathrm{AU}<a<0.80 \mathrm{AU}$ are chaotic and their eccentricities are excited to moderate and even high values, the characterized MMRs with respect to the accumulation or depletion of the asteroid belt are $3: 2(0.481 \mathrm{AU}), 2: 1$ (0.397 AU), 5:2 (0.342 AU), 4:3 (0.520 AU), 1:1 (0.630 AU), 2:3 (0.826 AU), 1:2 (1.000 $\mathrm{AU})$, and our results enrich those of Paper I for massless bodies over two consecutive 1000year intervals, showing a broader stable region beyond $0.80 \mathrm{AU}$. Note that there exist stable Trojan terrestrial bodies in a narrow stripe about 0.630 AU, involved in 1:1 MMR with Planet $\mathrm{D}$, and they can survive at least $10^{6} \mathrm{yr}$ with resulting small eccentricities in the extended integrations. The stable Trojan configurations may possibly appear in the extrasolar planetary systems (see also Dvorak 2008; Psychoyos \& Hadjidemetriou 2008 in this issue), e.g., Ji et al. (2005) explored such Trojan planets orbiting about 47 Uma, and Gozdziewski \& Konacki (2006) also argued that there may exist Trojan pair configurations in the HD 128311 and HD 82943 systems. Ford \& Gaudi (2006) developed a novel method of detecting Trojan companions to transiting close-in extrasolar planets and argue that the terrestrial-mass Trojans may be detectable with present ground-based observatories. Terrestrial Trojan planets with low eccentricity orbits close to 1 AU could potentially be habitable, and are worthy of further investigation in the future.

\section{Summary and Discussion}

In this work, we investigated the planetary configuration of three Neptune-mass companions similar to those surrounding HD 69830 and added one massive terrestrial planet in the region of $0.07 \mathrm{AU} \leqslant a \leqslant 1.20 \mathrm{AU}$ to examine the dynamical stability of terrestrial 
mass planets and to explore the asteroid structure in this system. We show that there are stable zones of at least $10^{5} \mathrm{yr}$ for the low-mass terrestrial planets located between 0.3 and $0.5 \mathrm{AU}$, and 0.8 and $1.2 \mathrm{AU}$ with final eccentricities of $e<0.20$. Moreover, we also find that the accumulation or depletion of the asteroid belt is also shaped by orbital resonances of the outer planets, for example, the asteroidal gaps of 2:1 and 3:2 MMRs with Planet C, and 5:2 and 1:2 resonances with Planet D. On the other hand, the stellar luminosity of HD 69830 is lower than that of the Sun, thus the HZ should shift inwards compared to our Solar System. In a dynamical consideration, the proper candidate regions for the existence of the potential terrestrial planets or HZs are $0.35 \mathrm{AU}<a<0.50$ $\mathrm{AU}$, and $0.80 \mathrm{AU}<a<1.00 \mathrm{AU}$ for relatively low eccentricities. Finally, we may summarize that the HD 69830 system can possess an asteroidal architecture resembling the Solar System and both the mean motion resonance (MMR) and secular resonances will work together to influence the distribution of the small bodies in the planetary system. In other simulations, we also show the potential Habitable zones for Earth-mass planets in the 47 UMa planetary system (see Ji et al. 2005), and the results imply that future space-based observations, e.g., CoRot, Kelper and TPF will hopefully produce a handful of samples belonging to the category of the terrestrial bodies.

\section{Acknowledgements}

We thank the anonymous referee for informative comments and suggestions that helped to improve the contents. This work is financially supported by the National Natural Science Foundations of China (Grants 10573040, 10673006, 10203005, 10233020) and the Foundation of Minor Planets of Purple Mountain Observatory.

\section{References}

Alibert, Y., et al. 2006, A\&A, 455, L25

Asghari, N., et al. 2004, Aछ A, 426, 353

Beichman, C. A., et al. 2005, ApJ, 626, 1061

Beichman, C. A., et al. 2006, ApJ, 652, 1674

Boss, A. P. 2006, ApJ, 644, L79

Butler, R. P., et al. 2006, ApJ, 646, 505

Dvorak, R. 2008, IAU S249, this issue

Ford, E. B., \&, Gaudi, B. S. 2006, ApJ, 652, L137

Gaidos, E., et al. 2007, Science, 318, 210

Gozdziewski, K., \& Konacki, M. 2006, ApJ, 647, 573

Gozdziewski, K, et al. 2007, ApJ, 657, 546

Ida, S., \& Lin, D. N. C. 2004, ApJ, 604, 388

Ji, J., Liu, L., Kinoshita, H., \& Li, G.Y. 2005, ApJ, 631, 1191

Ji, J., Kinoshita, H., Liu, L., \& Li, G.Y. 2007, ApJ, 657, 1092

Jones, B. W., Underwood, D. R., \& Sleep, P. N. 2005, ApJ, 622, 1091

Kasting, J. F., Whitmire, D. P., \& Reynolds, R. T. 1993, Icarus, 101, 108

Lovis, C., et al. 2006, Nature, 441, 305 (Paper I)

McArthur, B.E., et al. 2004, ApJ, 614, L81

Murray, C. D., \& Dermott, S. F. 1999, Solar System Dynamics (New York: Cambridge Univ. Press)

Psychoyos, D., \&, Hadjidemetriou, J. D. 2008, IAU S249, this issue

Raymond, S. N., Mandell, A. M., \& Sigurdsson, S. 2006, Science, 313, 1413

Rivera, E. J., et al. 2005, ApJ, 634, 625

Santos, N.C., et al. 2004, A\&A, 426, L19

von Bloh, W., Bounama, C., Cuntz, M., \& Franck, S. 2007, arXiv:0705.3758 